\title{
UNIVERSAL COMMENSURABILITY AUGMENTED TEICHMÜLLER SPACE AND MODULI SPACE
}

\author{
Guangming Hu, Hideki Miyachi and Yi Qi \\ Jinling Institute of Technology, College of Science \\ Nanjing, 211169, P. R. China; hgm326219@jit.edu.cn \\ Kanazawa University, College of Science and Engineering, School of Mathematics and Physics \\ Kakuma-machi, Kanazawa, Ishikawa, 920-1192, Japan; miyachi@se.kanazawa-u.ac.jp \\ Beihang University, School of Mathematics and Systems Science \\ Beijing, 100191, P. R. China; yiqi@buaa.edu.cn
}

\begin{abstract}
It is known that every unbranched finite covering $\alpha: \widetilde{S}_{g(\alpha)} \rightarrow S$ of a compact Riemann surface $S$ with genus $g \geq 2$ induces an isometric embedding $\Gamma_{\alpha}$ from the Teichmüller space $T(S)$ to the Teichüller space $T\left(\widetilde{S}_{g(\alpha)}\right)$. Actually, it has been showed that the isometric embedding $\Gamma_{\alpha}$ can be extended isometrically to the augmented Teichmüller space $\widehat{T}(S)$ of $T(S)$. Using this result, we construct a direct limit $\widehat{T}_{\infty}(S)$ of augmented Teichmüller spaces, where the index runs over all unbranched finite coverings of $S$. Then, we show that the action of the universal commensurability modular group $\operatorname{Mod}_{\infty}(S)$ can extend isometrically on $\widehat{T}_{\infty}(S)$. Furthermore, for any $X_{\infty} \in T_{\infty}(S)$, its orbit of the action of the universal commensurability $\operatorname{modular} \operatorname{group}_{\operatorname{Mod}}(S)$ on $\widehat{T}_{\infty}(S)$ is dense. Finally, we also construct a direct limit $\widehat{M}_{\infty}(S)$ of augmented moduli spaces by characteristic towers and show that the subgroup $\operatorname{Caut}\left(\pi_{1}(S)\right)$ of $\operatorname{Mod}_{\infty}(S)$ acts on $\widehat{T}_{\infty}(S)$ to produce $\widehat{M}_{\infty}(S)$ as the quotient.
\end{abstract}

\section{Introduction and main results}

Let $S$ be a closed orientable surface of genus $g \geq 2$. Let $T(S)$ be the Teichmüller space of marked hyperbolic structures on $S$. There is a natural complete metric $d_{T(S)}$ on $T(S)$, called the Teichmüller metric. The moduli space $M(S)$ is defined as the equivalent classes of $T(S)$ by the action of the modular $\operatorname{group} \operatorname{Mod}(S)$. A known compactification of $M(S)$ is called the Deligne-Mumford compactification, which is introduced in [6] by Deligne and Mumford. Abikoff introduced a partial compactification of $T(S)$, named the augmented Teichmüller space $\widehat{T}(S)$ [1, 2], whose orbit space $\widehat{M}(S)$ by the action of $\operatorname{Mod}(S)$ is called the augmented moduli space. It is known that the augmented moduli space is homeomorphic to the Deligne-Mumford compactification [7].

In this paper, we consider the related issues of augmented Teichmüller spaces and augmented moduli spaces respectively, which are divided into two parts as follows.

1.1. Universal commensurability augmented Teichmüller space. Let $\alpha: \widetilde{S}_{g(\alpha)} \rightarrow S$ be an unbranched finite covering of $S$, where $\widetilde{S}_{g(\alpha)}$ is a compact Riemann surface of genus $g(\alpha)$. It is known that the covering $\alpha$ can induce an isometrically (endowed with Teichmüller metrics) holomorphic embedding $\Gamma_{\alpha}: T(S) \rightarrow T\left(\widetilde{S}_{g(\alpha)}\right)$.

https://doi.org/10.5186/aasfm.2021.4660

2020 Mathematics Subject Classification: Primary 32G15, 30F60, 57M10.

Key words: Augmented Teichmüller space, commensurability modular group, augmented moduli space, characteristic tower.

This work is partially supported by NSFC Grant Number 11871085, 12001117, JSPS KAKENHI Grant Number 20H01800, 16H03933, 17H02843 and PhD research startup foundation of Jinling Institute Technology Grant Number jit-b-202011, jit-fhxm-2018. 
In [5], Biswas, Nag and Sullivan studied the directed system of Teichmüller spaces arising from these embeddings $\Gamma_{\alpha}: T(S) \rightarrow T\left(\widetilde{S}_{g(\alpha)}\right)$, as $\alpha$ runs over all finitely unbranched coverings of $S$. This direct limit of Teichmüller spaces, denoted by $T_{\infty}(S)$, is called the universal commensurability Teichmüller space and its completion is named the Teichmüller space for the universal hyperbolic solenoid (See $[12,13,14,15,16]$ for details of the solenoid). The universal commensurability Teichmüller space $T_{\infty}(S)$ carries a natural Weil-Petersson Kähler structure from scaling the Weil-Petersson pairing on each finite dimensional stratum. The universal commensurability Teichmüller space $T_{\infty}(S)$ has a biholomorphic automorphism group, called the universal commensurability modular group $\operatorname{Mod}_{\infty}(S)$.

In [5], the statement that the orbits of the action of $\operatorname{Mod}_{\infty}(S)$ on $T_{\infty}(S)$ are dense is actually equivalent to the following conjecture:

Ehrenpreis Conjecture. Let $S_{1}$ and $S_{2}$ be compact Riemann surfaces of genus at least two and $K>1$. Then there are compact Riemann surfaces $\widetilde{S}_{1}$ and $\widetilde{S}_{2}$ with unbranched finite coverings $\alpha_{1}: \widetilde{S}_{1} \rightarrow S_{1}$ and $\alpha_{2}: \widetilde{S}_{2} \rightarrow S_{2}$ and a $K$-quasiconformal mapping $\widetilde{f}: \widetilde{S}_{1} \rightarrow \widetilde{S}_{2}$.

In [10], Kahn and Markovic developed the notion of good pants homology and showed that it agrees with the standard homology on closed surfaces. Then they solved the Ehrenpreis Conjecture.

In [3], Biswas, Mitra and Nag proved that the action of $\operatorname{Mod}_{\infty}(S)$ can extend continuously on the direct limit of Thurston compactifications, and the orbits of the action of $\operatorname{Mod}_{\infty}(S)$ on the boundary of the direct limit of Thurston compactifications are dense.

In [8], it has been showed that the isometric embedding $\Gamma_{\alpha}$ can extend isometrically on the augmented Teichmüller space $\widehat{T}(S)$ of $T(S)$. So we naturally ask whether or not the results of Thurston compactifications can be generalized to augmented Teichmüller spaces. Here we have the following

Theorem 1.1. The action of the universal commensurability modular group $\operatorname{Mod}_{\infty}(S)$ can extend on the universal commensurability augmented Teichmüller space $\widehat{T}_{\infty}(S)$ isometrically, endowed with the Teichmüller metric.

Remark. Since the universal commensurability Teichmüller space $T_{\infty}(S)$ has a naturally complex manifold structure from each finite dimensional stratum, the universal commensurability modular group $\operatorname{Mod}_{\infty}(S)$, acting on $T_{\infty}(S)$, is a biholomorphic automorphism group. However, the augmented Teichmüller space has no manifold structure, hence $\widehat{T}_{\infty}(S)$ has no manifold structure and the element of $\operatorname{Mod}_{\infty}(S)$, acting on $\widehat{T}_{\infty}(S)$, is not holomorphic.

Combined with the Ehrenpreis conjecture, we have the following

Theorem 1.2. For any $X_{\infty} \in T_{\infty}(S)$, its orbit of the action of the universal commensurability modular group $\operatorname{Mod}_{\infty}(S)$ on the universal commensurability augmented Teichmüller space $\widehat{T}_{\infty}(S)$ is dense.

1.2. Universal commensurability augmented moduli space. Although the augmented Teichmüller space has no manifold structure, in [9], Hubbard and Koch obtained complex structures of augmented moduli spaces by the method of plumbing coordinates. In [4], Biswas and Nag introduced the characteristic covering 
of $S$ defined as follows: A covering $\alpha: \widetilde{S}_{g(\alpha)} \rightarrow S$ is called characteristic if every homeomorphism of $S$ lifts to one homeomorphism of $\widetilde{S}_{g(\alpha)}$. Then it has the following

Theorem 1.3. Any characteristic covering $\alpha$, from $\widetilde{S}_{g(\alpha)}$ to $S$, induces a holomorphic mapping $\widehat{\Phi}_{\alpha}: \widehat{M}(S) \rightarrow \widehat{M}\left(\widetilde{S}_{g(\alpha)}\right)$.

In [4], Biswas and Nag introduced a directed sub-system corresponding to characteristic tower coverings and showed that the subgroup Caut $\left(\pi_{1}(S)\right)$ of $\operatorname{Mod}_{\infty}(S)$ acts on $T_{\infty}(S)$ to produce the direct limit $M_{\infty}(S)$ of moduli spaces. In [3], Biswas, Mitra and Nag showed that Caut $\left(\pi_{1}(S)\right)$ acts on the direct limit of Thurston compactifications to produce the direct limit of Thurston compactified moduli spaces as the quotient. Similarly, we get the following

Theorem 1.4. The subgroup Caut $\left(\pi_{1}(S)\right)$ acts on the universal commensurability augmented Teichmüller space $\widehat{T}_{\infty}(S)$ to produce the direct limit $\widehat{M}_{\infty}(S)$ of augmented moduli spaces as the quotient.

\section{Preliminaries}

2.1. Augmented Teichmüller space. A marked Riemann surface modeled on $S$ is defined by one tuple $(R, f)$, where $R$ is a compact Riemann surface and $f: S \rightarrow R$ is a quasiconformal mapping. Two marked Riemann surfaces $\left(R_{1}, f_{1}\right)$ and $\left(R_{2}, f_{2}\right)$ are Teichmüller equivalent if there exists a conformal mapping $h: R_{1} \rightarrow R_{2}$ such that $h$ is homotopic to $f_{2} \circ f_{1}^{-1}$. The Teichmüller space $T(S)$ can be defined as

$T(S)=\{X:=[R, f] \mid(R, f)$ is a marked Riemann surface modeled on $S\}$,

where $X$ is the Teichmüller equivalent class containing $(R, f)$.

There is a natural complete metric $d_{T(S)}$ on $T(S)$, called Teichmüller metric, which is defined as

$$
d_{T(S)}\left(X_{1}, X_{2}\right)=\frac{1}{2} \inf \{\log K(h)\}, \quad \text { for any } X_{1}, X_{2} \in T(S),
$$

where the infimum takes over all quasiconformal mappings $h: R_{1} \rightarrow R_{2}$ homotopic to $f_{2} \circ f_{1}^{-1}$ and $K(h)$ is the maximal dilatation of $h$.

Denote by $\mathcal{S}$ the set of all homotopic classes of non-trivial simple closed curves on $S$ and $[\gamma]$ represents the element of $\mathcal{S}$. Let $\mathcal{A}=\left\{\gamma_{1}, \cdots, \gamma_{n}\right\}$ be a multicurve on $S$. A multicurve is maximal on $S$ if the multicurve has $3 g-3$ components. Let $S^{\mathcal{A}}=\bigcup_{i=1}^{k_{n}} S^{i}$ be the surface which contracts the multicurve $\mathcal{A}$ of $S$ to points and is a homeomorphism except $\mathcal{A}$. Let $T_{S \mathcal{A}}$ be the product of Teichmüller spaces of the components. The augmented Teichmüller space $\widehat{T}(S)$ of $S$ is composed by the disjoint union of strata $T_{S \mathcal{A}}$. The minimal strata, which correspond to maximal multicurves, are points.

There is a natural metric $d_{\widehat{T}(S)}$ on the augmented Teichüller space $\widehat{T}(S)$. For any $\dot{X}_{1}, \dot{X}_{2} \in \widehat{T}(S)$, if they are in the same stratum, the distance is defined as

$$
d_{\widehat{T}(S)}\left(\dot{X}_{1}, \dot{X}_{2}\right)=\max _{i=1, \ldots, k_{n}}\left\{d_{T\left(S^{i}\right)}\left(X_{1}^{i}, X_{2}^{i}\right)\right\}, \quad \text { for } X_{1}^{i}, X_{2}^{i} \in T\left(S^{i}\right) .
$$

Otherwise,

$$
d_{\widehat{T}(S)}\left(\dot{X}_{1}, \dot{X}_{2}\right)=+\infty
$$

2.2. Universal commensurability Teichmüller space. Let $\alpha: \widetilde{S}_{g(\alpha)} \rightarrow S$ be an unbranched finite covering of $S$. Construct a category $\mathcal{C}(S)$ of some topological objects and morphisms as follows: the objects, denoted by $\operatorname{Ob}(\mathcal{C}(S))$, constitute all 
unbranched finite covering surfaces of $S$ equipped with a base point $(\star)$ and the morphisms, denoted by $\operatorname{Mor}(\mathcal{C}(S))$, are based homotopic classes of pointed covering mappings $\alpha: \widetilde{S}_{g(\alpha)} \rightarrow S$. The monomorphism of fundamental groups induced by any representative of the based homotopic class of coverings $\alpha$ is unambiguously defined. For two morphisms $\alpha, \beta \in \operatorname{Mor}(\mathcal{C}(S))$, we say $\alpha \prec \beta$ if there is a commuting triangle of morphisms $\beta=\alpha \circ \theta$, where $\theta$ is a unbranched finite covering. Since we are working with surfaces with based points, the factoring morphism is uniquely determined. Therefore there exists a partially ordering relation in $\operatorname{Mor}(\mathcal{C}(S))$ by factorizations of morphisms. It is showed in [5] that the morphisms $\operatorname{Mor}(\mathcal{C}(S))$ constitute a directed set under the partially ordering relation.

It is known that a morphism $\alpha$ can induce an isometric (endowed with Teichmüller metrics) embedding $\Gamma_{\alpha}: T(S) \rightarrow T\left(\widetilde{S}_{g(\alpha)}\right)$. Similar to the construction of the above category, we can create a category $\mathcal{C}(T(S))$ of Teichmüller spaces and isometric embeddings as follows: the objects, denoted by $\mathrm{Ob}(\mathcal{C}(T(S)))$, constitute all Teichmüller spaces induced by unbranched finite coverings of $S$ and the morphisms, denoted by $\operatorname{Mor}(\mathcal{C}(T(S)))$, are all isometric embeddings $\Gamma_{\alpha}: T(S) \rightarrow T\left(\widetilde{S}_{g(\alpha)}\right)$ for $\alpha \in \mathcal{C}(S)$. For each $\alpha \prec \beta$, we have the corresponding isometric embedding $\Gamma_{\theta}$, satisfied with $\Gamma_{\beta}=\Gamma_{\theta} \circ \Gamma_{\alpha}$, where $\beta=\alpha \circ \theta$. There exists a partially ordering relation in $\mathcal{C}(T(S))$ by factorizations of isometric embeddings. Then the morphisms $\operatorname{Mor}(\mathcal{C}(T(S)))$ produce a natural directed system induced by $\operatorname{Mor}(\mathcal{C}(S))$.

From this directed system, the direct limit Teichmüller space over $S$ is defined as $T_{\infty}(S):=\operatorname{dir} . \lim . T\left(\widetilde{S}_{g(\alpha)}\right)$ for $\alpha \in \operatorname{Mor}(\mathcal{C}(S))$ which is named the universal commensurability Teichmüller space. It is an inductive limit of finite dimensional spaces [17].

The Teichmüller metric $d_{T_{\infty}(S)}$ on $T_{\infty}(S)$ is defined as the Teichmüller metric $d_{T\left(\widetilde{S}_{g(\alpha)}\right)}$ on every stratification $T\left(\widetilde{S}_{g(\alpha)}\right)$ for $\alpha \in \operatorname{Mor}(\mathcal{C}(S))$.

2.3. Universal commensurability modular group. It is showed in [5] that every morphism $\alpha: \widetilde{S}_{g(\alpha)} \rightarrow S$ can induce a natural Teichmüller metric preserving homeomorphism $\Gamma_{\infty}(\alpha): T_{\infty}\left(\widetilde{S}_{g(\alpha)}\right) \rightarrow T_{\infty}(S)$ because the directed set $\operatorname{Mor}\left(\mathcal{C}\left(\widetilde{S}_{g(\alpha)}\right)\right)$ is cofinal in $\operatorname{Mor}(\mathcal{C}(S))$.

For a given pair of morphisms

$$
\alpha: \widetilde{S} \rightarrow S \text { and } \beta: \widetilde{S} \rightarrow S,
$$

we have an isometric automorphism $\Gamma_{\infty}(\beta) \circ \Gamma_{\infty}^{-1}(\alpha)$ on $T_{\infty}(S)$. More generally, we are given a cycle of morphisms starting and ending at $S$ as follows:

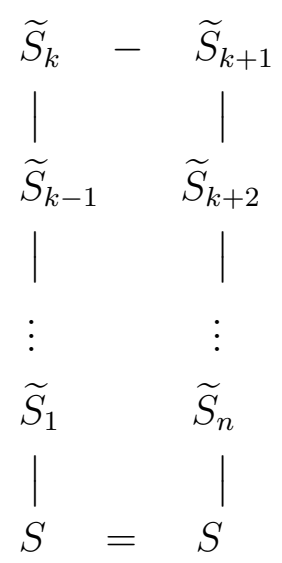


where $\widetilde{S}_{i}, S$ are all objects of the category $\mathcal{C}(S)$ and all horizontal and vertical lines represent morphisms of $\mathcal{C}(S)$. Since $\Gamma_{\infty}(\alpha)$ is invertible, the horizontal and the vertical lines in the above cycle of morphisms are allowed in any direction. Thus we can define an isometric automorphism $\varphi: T_{\infty}(S) \rightarrow T_{\infty}(S)$ which is a composition around the undirect cycle of morphisms starting from $S$ and returning to $S$. The group $\operatorname{Mod}_{\infty}(S)$ consists of all thus automorphism, called the universal commensurability modular group, acting on $T_{\infty}(S)$ isometrically.

2.4. Complex structure of augmented moduli space. The modular $\operatorname{group} \operatorname{Mod}(S)$ is defined as the group of homotopic equivalent classes of orientationpreserving homeomorphisms of $S$. Two orientation preserving homeomorphisms $\phi, \varphi: S \rightarrow S$ are equivalent if $\phi \circ \varphi^{-1}$ is isotopic to the identity map on $S$. Since $\operatorname{Mod}(S)$ acts on $\left(T(S), d_{T(S)}\right)$ discretely and isometrically, the moduli space $M(S)$ is defined as the equivalent classes of $T(S)$ by the action of $\operatorname{Mod}(S)$. Let $\mathcal{X}:=\{R, f\}$ be represented as the element of $M(S)$. The orbit space $\widehat{M}(S)=\widehat{\operatorname{Teich}}(S) / \operatorname{Mod}(S)$ is called the augmented moduli space and is compact with the quotient topology [2]. Denote by $\dot{\mathcal{X}}$ the element of $\widehat{M}(S)$.

In [9], Hubbard and Koch introduced some subgroups of the modular group $\operatorname{Mod}(S)$ as follows:

Definition 2.1. Denote by $\mathcal{A}$ a multicurve on $S$. Let $S / \mathcal{A}$ be the topological surface obtained from collapsing the elements $\mathcal{A}$ to points. The subgroup $\Delta_{\mathcal{A}}(S)$ of $\operatorname{Mod}(S)$ is defined as the group generated by Dehn twists around the Teichmüller space.

Consider the space

$$
U_{\mathcal{A}}(S):=\bigcup_{\mathcal{A}^{\prime} \subseteq \mathcal{A}} T_{S^{\mathcal{A}^{\prime}}} \subseteq \widehat{T}(S),
$$

and the subgroup $\Delta_{\mathcal{A}}(S)$ of $\operatorname{Mod}(S)$ acts on $U_{\mathcal{A}}(S)$. The space

$$
\mathcal{Q}_{\mathcal{A}}(S):=U_{\mathcal{A}}(S) / \Delta_{\mathcal{A}}(S)
$$

is the quotient topology inherited from $\widehat{T}(S)$. The stratum of $\mathcal{Q}_{\mathcal{A}}(S)$ is defined as $T_{S^{\mathcal{A}^{\prime}}} / \Delta_{\mathcal{A}}(S)$, denoted by $\mathcal{Q}_{\mathcal{A}}^{\mathcal{A}^{\prime}}(S)$.

If complete $\mathcal{A}$ to a maximal multicurve $\mathcal{A}_{\text {max }}$, the Fenchel-Nielsen coordinate of $\mathcal{Q}_{\mathcal{A}}(S)$ represents $\left(\mathbf{R}_{+} \times \mathbf{R}\right)^{\mathcal{A}_{\max }-\mathcal{A}} \times \mathbf{C}^{\mathcal{A}}[9]$.

For $p \in \mathcal{Q}_{\mathcal{A}}^{\mathcal{A}}(S)$, there exists an open neighborhood $N$ of $p$ in $\mathcal{Q}_{\mathcal{A}}^{\mathcal{A}}(S)$. Let $\mathcal{P}_{\mathcal{A}}(S)=$ $N \times \mathbf{D}^{\mathcal{A}}$ be a complex manifold and it is the union of strata

$$
\mathcal{P}_{\mathcal{A}}(S)=\bigcup_{\mathcal{A}^{\prime} \subset \mathcal{A}} \mathcal{P}_{\mathcal{A}}^{\mathcal{A}^{\prime}}(S)
$$

where

$$
\mathcal{P}_{\mathcal{A}}^{\mathcal{A}^{\prime}}(S)=\left\{(p, \mathbf{z}) \in N \times \mathbf{D}^{\mathcal{A}} \mid z_{\gamma}=0 \Leftrightarrow \gamma \in \mathcal{A}^{\prime}\right\} .
$$

The Fenchel-Nielsen coordinate of $\mathcal{P}_{\mathcal{A}}(S)$ is defined as

$$
\left(l_{\gamma}, \tau_{\gamma}\right), \gamma \in \mathcal{A}_{\max }-\mathcal{A} ; \quad l_{\gamma} e^{2 \pi i \tau_{\gamma} / l_{\gamma}}, \gamma \in \mathcal{A}
$$

and it can define a mapping $\Psi: \mathcal{P}_{\mathcal{A}}(S) \rightarrow \mathcal{Q}_{\mathcal{A}}(S)$. Hubbard and Koch proved the following result:

Lemma 2.2. For any $(p, \mathbf{z}) \in \mathcal{P}_{\mathcal{A}}(S)$ with $\|\mathbf{z}\|$ sufficiently small, there exists a neighborhood $V^{\prime}$ such that $V:=\Psi\left(V^{\prime}\right)$ is an open set in $\mathcal{Q}_{\mathcal{A}}(S)$ and $\Psi: V^{\prime} \rightarrow V$ is a homeomorphism. 
From Lemma 2.2, they constructed the complex structure of $\mathcal{Q}_{\mathcal{A}}(S)$ as follows:

Theorem A. For every multicurve $\mathcal{A}$ on $S$, there exists a complex manifold structure on $\mathcal{Q}_{\mathcal{A}}(S)$.

Since

$$
\widehat{M}(S)=\bigcup_{\mathcal{A} \subset \mathcal{S}}\left(\mathcal{Q}_{\mathcal{A}}(S) / \operatorname{Mod}(S)\right),
$$

they obtained the following

Theorem B. The augmented moduli space $\widehat{M}(S)$ has an analytic orbifold Structure.

2.5. Universal commensurability moduli space. A morphism $\alpha: \widetilde{S}_{g(\alpha)} \rightarrow S$ is called characteristic if the fundamental group $\pi_{1}\left(\widetilde{S}_{g(\alpha)}\right)$ is a characteristic subgroup of the fundamental group $\pi_{1}(S)$. In other words, the subgroup $\pi_{1}\left(\widetilde{S}_{g(\alpha)}\right) \subseteq \pi_{1}(S)$ must be invariant by every automorphism of $\pi_{1}(S)$. This can yield a homomorphism: $L_{\alpha}: \operatorname{Aut}\left(\pi_{1}(S)\right) \rightarrow \operatorname{Aut}\left(\pi_{1}\left(\widetilde{S}_{g(\alpha)}\right)\right)$. The topological characterization of a characteristic cover is that every homeomorphism of $S$ lifts to one homeomorphism of $\widetilde{S}_{g(\alpha)}$, and the homomorphism $L_{\alpha}$ corresponds to this lifting process. The characteristic subgroups of finite index form a cofinal family among all subgroups of finite index in $\pi_{1}(S)$ [4].

Consider the characteristic tower $\operatorname{Mor}\left(\mathcal{C}^{c h}(S)\right)$ over $S$ consisting of only characteristic morphisms. For $\alpha, \beta \in \operatorname{Mor}\left(\mathcal{C}^{c h}(S)\right)$, we say $\alpha \prec \beta$ if and only if $\beta=\alpha \circ \theta$ which $\theta$ is also a characteristic morphism. The characteristic tower $\operatorname{Mor}\left(\mathcal{C}^{c h}(S)\right)$ is a directed set under the partial ordering given by factorization of characteristic morphisms. It is known that the characteristic tower $\operatorname{Mor}\left(\mathcal{C}^{c h}(S)\right)$ is the cofinal subset of $\operatorname{Mor}(\mathcal{C}(S))$ [4]. Any characteristic morphism $\alpha$, from $\widetilde{S}_{g(\alpha)}$ to $S$, induces a morphism $\Phi_{\alpha}: M(S) \rightarrow M\left(\widetilde{S}_{g(\alpha)}\right)$ which is an algebraic morphism between these normal quasi-projective varieties. Similarly, we have a direct limit $M_{\infty}(S)$ of moduli spaces over $S$ as follows:

$$
M_{\infty}(S):=\operatorname{dir} . \lim . M\left(\widetilde{S}_{g(\alpha)}\right), \quad \alpha \in \operatorname{Mor}\left(\mathcal{C}^{c h}(S)\right),
$$

which is called the universal commensurability moduli space.

Using the homomorphisms $L_{\alpha}: \operatorname{Aut}\left(\pi_{1}(S)\right) \rightarrow \operatorname{Aut}\left(\pi_{1}\left(\widetilde{S}_{g(\alpha)}\right)\right)$, we can define a direct limit of automorphism groups over $S$ as follows:

$$
\operatorname{Caut}\left(\pi_{1}(S)\right)=\operatorname{dir} \cdot \lim \cdot \operatorname{Aut}\left(\pi_{1}\left(\widetilde{S}_{g(\alpha)}\right), \quad \alpha \in \operatorname{Mor}\left(\mathcal{C}^{c h}(S)\right) .\right.
$$

\section{Universal commensurability augmented Teichmüller space and universal commensurability modular group}

In this section, we give the definition of the universal commensurability augmented Teichüller space over $S$. Then we consider the action of the universal commensurability modular group on the universal commensurability augmented Teichüller space.

The following lemma [8] plays an important role in defining the universal commensurability augmented Teichüller space.

Lemma 3.1. Let $\alpha: \widetilde{S}_{g(\alpha)} \rightarrow S$ be an unbranched finite covering of a compact Riemann surface $S$ with genus $g \geq 2$. Then the isometric embedding $\Gamma_{\alpha}$ can extend to $\widehat{\Gamma}_{\alpha}: \widehat{T}(S) \rightarrow \widehat{T}\left(\widetilde{S}_{g(\alpha)}\right)$ isometrically. 
Similar to the universal commensurability Teichmüller space, we can create a category $\mathcal{C}(\widehat{T}(S))$ of augmented Teichmüller spaces and isometric embeddings as follows: the objects, denoted by $\operatorname{Ob}(\mathcal{C}(\widehat{T}(S)))$, constitute all augmented Teichmüller spaces induced by unbranched finite coverings of $S$ and the morphisms, denoted by $\operatorname{Mor}(\mathcal{C}(\widehat{T}(S)))$, are all isometric embeddings $\widehat{\Gamma}_{\alpha}: \widehat{T}(S) \rightarrow \widehat{T}\left(\widetilde{S}_{g(\alpha)}\right)$. For each $\alpha \prec \beta$, we have the corresponding isometric embedding $\widehat{\Gamma}_{\theta}$, satisfied with $\widehat{\Gamma}_{\beta}=\widehat{\Gamma}_{\theta} \circ \widehat{\Gamma}_{\alpha}$, where $\beta=\alpha \circ \theta$. There exists a partially ordering relation in $\operatorname{Mor}(\mathcal{C}(\widehat{T}(S)))$ by factorizations of isometric embeddings. The morphisms $\operatorname{Mor}(\mathcal{C}(S))$ constitute a directed set under the partially ordering relation. Then the morphisms $\operatorname{Mor}(\mathcal{C}(\widehat{T}(S)))$ produce a natural directed system induced by $\operatorname{Mor}(\mathcal{C}(S))$.

Definition 3.2. For the directed system $\operatorname{Mor}(\mathcal{C}(\widehat{T}(S)))$, the direct limit of augmented Teichmüller spaces over $S$ is defined as

$$
\widehat{T}_{\infty}(S):=\operatorname{dir} . \lim . \widehat{T}\left(\widetilde{S}_{g(\alpha)}\right), \quad \alpha \in \operatorname{Mor}(\mathcal{C}(S))
$$

which is called the universal commensurability augmented Teichmüller space.

Definition 3.3. The Teichmüller metric $d_{\widehat{T}_{\infty}(S)}$ on $\widehat{T}_{\infty}(S)$ is defined as the Teichmüller metric $d_{\widehat{T}\left(\widetilde{S}_{g(\alpha)}\right)}$ on every stratification $\widehat{T}\left(\widetilde{S}_{g(\alpha)}\right)$ for $\alpha \in \operatorname{Mor}(\mathcal{C}(S))$.

Next, we show that the action of the universal commensurability modular group $\operatorname{Mod}_{\infty}(S)$ is isometric, endowed with the Teichmüller metric, on $\widehat{T}_{\infty}(S)$.

Proof of Theorem 1.1. Suppose that $\alpha: \widetilde{S}_{g(\alpha)} \rightarrow S$ is a morphism, then it can induce a natural Teichmüller metric preserving homeomorphism $\Gamma_{\infty}(\alpha): T_{\infty}\left(\widetilde{S}_{g(\alpha)}\right) \rightarrow$ $T_{\infty}(S)$. According to the definition of $T_{\infty}\left(\widetilde{S}_{g(\alpha)}\right)$, it is easy to know any stratification $T\left(\widetilde{S}_{g(\delta)}\right), \delta \in \operatorname{Mor}\left(\mathcal{C}\left(\widetilde{S}_{g(\alpha)}\right)\right)$, of $T_{\infty}\left(\widetilde{S}_{g(\alpha)}\right)$ can be embedded in some stratification $T\left(S_{g(\eta)}\right), \eta \in \operatorname{Mor}(\mathcal{C}(S))$, of $T_{\infty}(S)$ isometrically. By Lemma 3.1, the isomeric embedding can extend to the corresponding augmented Teichmüller space isometrically, then we have a natural Teichmüller metric preserving embedding $\widehat{\Gamma}_{\infty}(\alpha): \widehat{T}_{\infty}\left(\widetilde{S}_{g(\alpha)}\right) \rightarrow$ $\widehat{T}_{\infty}(S)$ by the definition of the universal commensurability augmented Teichmüller space.

Since $\Gamma_{\infty}(\alpha): T_{\infty}\left(\widetilde{S}_{g(\alpha)}\right) \rightarrow T_{\infty}(S)$ is a homeomorphism, we have the invertible mapping $\Gamma_{\infty}^{-1}(\alpha): T_{\infty}(S) \rightarrow T_{\infty}\left(\widetilde{S}_{g(\alpha)}\right)$. Then any stratification $T\left(S_{g(\eta)}\right), \eta \in$ $\operatorname{Mor}(\mathcal{C}(S))$, of $T_{\infty}(S)$ also can be embedded in some stratification $T\left(\widetilde{S}_{g(\delta)}\right), \delta \in$ $\operatorname{Mor}(\mathcal{C}(\widetilde{S}))$, of $T_{\infty}(\widetilde{S})$ isometrically. By Lemma 3.1 again, the isomeric embedding can extend to the corresponding augmented Teichmüller space isometrically, hence $\widehat{\Gamma}_{\infty}(\alpha): \widehat{T}_{\infty}\left(\widetilde{S}_{g(\alpha)}\right) \rightarrow \widehat{T}_{\infty}(S)$ is surjective. Here we show that $\alpha$ can induce a natural Teichmüller metric preserving homeomorphism $\widehat{\Gamma}_{\infty}(\alpha): \widehat{T}_{\infty}\left(\widetilde{S}_{g(\alpha)}\right) \rightarrow \widehat{T}_{\infty}(S)$.

For a given pair of morphisms

$$
\alpha: \widetilde{S} \rightarrow S \text { and } \beta: \widetilde{S} \rightarrow S
$$

there exists a natural isometric automorphism $\widehat{\Gamma}_{\infty}(\beta) \circ \widehat{\Gamma}_{\infty}^{-1}(\alpha)$ on $\widehat{T}_{\infty}(S)$. Since any element $\varphi \in \operatorname{Mod}_{\infty}(S)$ can be induced by the following cycle of morphisms starting 
and ending at $S$

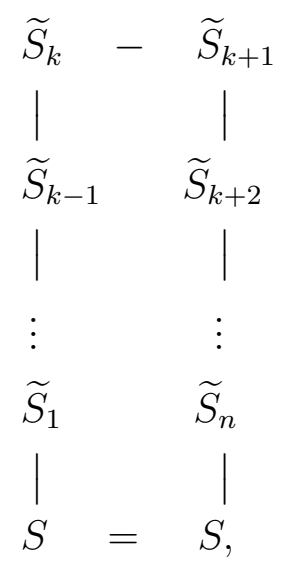

$\varphi$ can extend on the universal commensurability augmented Teichmüller space $\widehat{T}_{\infty}(S)$ isometrically by the above method. Therefore the action of the $\operatorname{group}_{\operatorname{Mod}}(S)$ can extend on $\widehat{T}_{\infty}(S)$ isometrically.

Proof of Theorem 1.2. Since the Ehrenpreis Conjecture is affirmatively solved, we have that the orbits of the action of $\operatorname{Mod}_{\infty}(S)$ on $T_{\infty}(S)$ are dense. Namely, for any $X_{\infty} \in T_{\infty}(S)$, its orbit of the action of the universal commensurability modular group $\operatorname{Mod}_{\infty}(S)$ on $T_{\infty}(S)$ is dense. For any $\dot{X}_{\infty} \in \widehat{T}_{\infty}(S)$, there exists a sequence $\left\{X_{\infty}^{n}\right\}_{n=1}^{\infty} \subseteq T_{\infty}(S)$ converging to $\dot{X}_{\infty}$. Since the orbits of the action of $\operatorname{Mod}_{\infty}(S)$ on $T_{\infty}(S)$ are dense, for any $X_{\infty}^{n}$, there is a sequence $\left\{X_{\infty}^{n, m}\right\}_{m=1}^{\infty}$ in the orbit of $X_{\infty}$ converging to it. Then we can choose a sequence $\left\{X_{\infty}^{n, n}\right\}_{n=1}^{\infty}$ converging to $\dot{X}_{\infty}$ by the Cantor diagonal method. Therefore, the orbit of $X_{\infty}$ on the universal commensurability augmented Teichmüller space $\widehat{T}_{\infty}(S)$ is dense.

\section{Universal commensurability augmented moduli space}

In this section, we show that any characteristic covering $\alpha$, from $\widetilde{S}_{g(\alpha)}$ to $S$, induces an analytic mapping $\widehat{\Phi}_{\alpha}: \widehat{M}(S) \rightarrow \widehat{M}\left(\widetilde{S}_{g(\alpha)}\right)$. Then we construct the universal commensurability augmented moduli space and show that the $\operatorname{subgroup} \operatorname{Caut}\left(\pi_{1}(S)\right)$ acts on the universal commensurability augmented Teichmüller space $\widehat{T}_{\infty}(S)$ to produce the direct limit $\widehat{M}_{\infty}(S)$ of augmented moduli spaces as the quotient.

Let $\alpha: \widetilde{S}_{g(\alpha)} \rightarrow S$ be a characteristic covering of a compact Riemann surface $S$ with genus $g \geq 2$. For any multicurve $\mathcal{A}$ on $S$, let $\widetilde{\mathcal{A}}_{g(\alpha)}:=\alpha^{-1}(\mathcal{A})$ be the preimage on $\widetilde{S}_{g(\alpha)}$ of $\mathcal{A}$.

Lemma 4.1. Let $\alpha: \widetilde{S}_{g(\alpha)} \rightarrow S$ be a characteristic covering of a compact Riemann surface $S$ with genus $g \geq 2$. For every multicurve $\mathcal{A}$ on $S$, then there exists a holomorphic mapping $\mathcal{E}_{\alpha}: \mathcal{Q}_{\mathcal{A}}(S) \rightarrow \mathcal{Q}_{\widetilde{\mathcal{A}}_{g(\alpha)}}\left(\widetilde{S}_{g(\alpha)}\right)$.

Proof. From Lemma 3.1, there exists an isometric embedding $\widehat{\Gamma}_{\alpha}: U_{\mathcal{A}}(S) \rightarrow$ $U_{\widetilde{\mathcal{A}}_{g(\alpha)}}\left(\widetilde{S}_{g(\alpha)}\right)$. Since the covering mapping $\alpha$ can induce a monomorphism from the subgroup $\Delta_{\mathcal{A}}(S)$ of $\operatorname{Mod}(S)$ to the subgroup $\Delta_{\widetilde{\mathcal{A}}_{g(\alpha)}}\left(\widetilde{S}_{g(\alpha)}\right)$ of $\operatorname{Mod}\left(\widetilde{S}_{g(\alpha)}\right)$, the isometric embedding $\widehat{\Gamma}_{\alpha}: U_{\mathcal{A}}(S) \rightarrow U_{\widetilde{\mathcal{A}}_{g(\alpha)}}\left(\widetilde{S}_{g(\alpha)}\right)$ can induce a continuous mapping $\mathcal{E}_{\alpha}: \mathcal{Q}_{\mathcal{A}}(S) \rightarrow \mathcal{Q}_{\widetilde{\mathcal{A}}_{g(\alpha)}}\left(\widetilde{S}_{g(\alpha)}\right)$. 
For any point $p$ of $\mathcal{Q}_{\mathcal{A}}^{\mathcal{A}}(S)$, there exists a point $\widetilde{p}:=\mathcal{E}_{\alpha}(p)$ in $\mathcal{Q}_{\widetilde{\mathcal{A}}_{g(\alpha)}}^{\widetilde{\mathcal{S}}_{g(\alpha)}}\left(\widetilde{S}_{g(\alpha)}\right)$. According to Lemma 2.2, there exists a neighborhood $\widetilde{V}_{1 *} \subset \mathcal{P}_{\mathcal{A}}\left(\widetilde{S}_{g(\alpha)}\right)$ such that $\widetilde{V}_{1}=\widetilde{\Psi}\left(\widetilde{V}_{1 *}\right)$ is an open set of $\widetilde{p}$ in $\mathcal{Q}_{\mathcal{A}}\left(\widetilde{S}_{g(\alpha)}\right)$ and $\widetilde{\Psi}: \widetilde{V}_{1 *} \rightarrow \widetilde{V}_{1}$ is a homeomorphism. Since $\mathcal{E}_{\alpha}$ is a continuous mapping, $V_{1}=\mathcal{E}_{\alpha}^{-1}\left(\widetilde{V}_{1}\right)$ is an open set in $\mathcal{Q}_{\mathcal{A}}(S)$. Using Lemma 2.2 again, there exists a neighborhood $V_{2 *} \subset \mathcal{P}_{\mathcal{A}}(S)$ such that $V_{2}=\Psi\left(V_{2 *}\right)$ is an open set in $\mathcal{Q}_{\mathcal{A}}(S)$ and $\Psi: V_{2 *} \rightarrow V_{2}$ is a homeomorphism. Then there exists a continuous mapping $\widetilde{\Psi}^{-1} \circ \mathcal{E}_{\alpha} \circ \Psi: \Psi^{-1}\left(V_{1} \cap V_{2}\right) \rightarrow \widetilde{\Psi}^{-1} \circ \mathcal{E}_{\alpha}\left(V_{1} \cap V_{2}\right)$, where $\Psi^{-1}\left(V_{1} \cap V_{2}\right) \subset \mathcal{P}_{\mathcal{A}}(S)=N \times \mathbf{D}^{\mathcal{A}}$ and $\widetilde{\Psi}^{-1} \circ \mathcal{E}_{\alpha}\left(V_{1} \cap V_{2}\right) \subset \mathcal{P}_{\widetilde{\mathcal{A}}_{g(\alpha)}}\left(\widetilde{S}_{g(\alpha)}\right)=\widetilde{N}_{g(\alpha)} \times \mathbf{D}^{\widetilde{\mathcal{A}}_{g(\alpha)}}$ are complex sub-manifolds. We know that $N \subset T_{S^{\mathcal{A}}}$ and $\widetilde{N}_{g(\alpha)} \subset T_{\widetilde{S}_{\tilde{\mathcal{A}}_{g(\alpha)}}}$ are two products of Teichmüller spaces of the components. It is showed in [11] that there exists a holomorphic embedding from every factor of $T_{S \mathcal{A}}$ to the corresponding factor of $T_{\widetilde{S}^{\tilde{A}(\alpha)}}$ by $\alpha: \widetilde{S}_{g(\alpha)} \rightarrow S$. For $\gamma \in \mathcal{A}$, the factor of is $z_{\gamma}=l_{\gamma} e^{2 \pi i \tau_{\gamma} / l_{\gamma}} \in \mathbf{D}$. Then the factor of one component $\widetilde{\gamma}$ of $\alpha^{-1}(\gamma)$ can be represented as $z_{\widetilde{\gamma}}=\widetilde{l}_{\gamma} e^{2 \pi i \widetilde{\tau}_{\gamma} / \widetilde{l}_{\gamma}}=$ $m l_{\gamma} e^{2 \pi i m \tau_{\gamma} /\left(m l_{\gamma}\right)}=m z_{\gamma}$ in a neighborhood of the original point, where $m$ is a positive integer. Therefore, $\widetilde{\Psi}^{-1} \circ \mathcal{E}_{\alpha} \circ \Psi: \Psi^{-1}\left(V_{1} \cap V_{2}\right) \rightarrow \widetilde{\Psi}^{-1} \circ \mathcal{E}_{\alpha}\left(V_{1} \cap V_{2}\right)$ is a holomorphic mapping.

For any point $p$ of $\mathcal{Q}_{\mathcal{A}}(S)-\mathcal{Q}_{\mathcal{A}}^{\mathcal{A}}(S)$, it belongs to some stratum $\mathcal{Q}_{\mathcal{A}}^{\mathcal{A}^{\prime}}(S)$. According to Theorem $A$, there is a holomorphic map $\mathcal{P}_{\mathcal{A}}^{\mathcal{A}^{\prime}}: \mathcal{Q}_{\mathcal{A}^{\prime}}(S) \rightarrow \mathcal{Q}_{\mathcal{A}}(S)$, which consists of quotienting by $\Delta_{\mathcal{A}-\mathcal{A}^{\prime}}(S)$. Since the curves of $\mathcal{A}-\mathcal{A}^{\prime}$ are not collapsed at $p$, the curves of $\widetilde{\mathcal{A}}_{g(\alpha)}-\widetilde{\mathcal{A}}_{g(\alpha)}^{\prime}$ are not collapsed at $\widetilde{p}:=\mathcal{E}_{\alpha}(p)$. Choose as a local chart at $p$ a section of $\mathcal{P}_{\mathcal{A}}^{\mathcal{A}^{\prime}}$ over a neighborhood of $p$ contained in $\mathcal{Q}_{\mathcal{A}}(S)$. Let $p^{\prime}$ be a preimage of $p$ in the section of $\mathcal{P}_{\mathcal{A}}^{\mathcal{A}^{\prime}}$. Similar to the above analysis, there exist two neighborhoods $V_{1}$ and $V_{2}$ of $p$ in $\mathcal{Q}_{\mathcal{A}}(S)$ such that $\widetilde{\Psi}^{\prime} \circ\left(\mathcal{P}_{\widetilde{\mathcal{A}}_{g(\alpha)}}^{\widetilde{\mathcal{A}}_{g(\alpha)}^{\prime}}\right)^{-1} \circ \mathcal{E}_{\alpha} \circ \mathcal{P}_{\mathcal{A}}^{\mathcal{A}^{\prime}} \circ\left(\Psi^{\prime}\right)^{-1}$ : $\Psi^{\prime} \circ\left(\mathcal{P}_{\mathcal{A}}^{\mathcal{A}^{\prime}}\right)^{-1}\left(V_{1} \cap V_{2}\right) \rightarrow \widetilde{\Psi}^{\prime} \circ\left(\mathcal{P}_{\widetilde{\mathcal{A}}_{g(\alpha)}}^{\widetilde{\mathcal{A}}_{g(\alpha)}^{\prime}}\right)^{-1} \circ \mathcal{E}_{\alpha}\left(V_{1} \cap V_{2}\right)$ is a holomorphic mapping, where $\left(\mathcal{P}_{\widetilde{\mathcal{A}}_{g(\alpha)}^{(\alpha)}}^{\widetilde{\mathcal{A}}_{g}^{\prime}}\right)^{-1} \circ \mathcal{E}_{\alpha} \circ \mathcal{P}_{\mathcal{A}}^{\mathcal{A}^{\prime}}: \mathcal{Q}_{\mathcal{A}^{\prime}}(S) \rightarrow \mathcal{Q}_{\widetilde{\mathcal{A}}_{g(\alpha)}^{\prime}}\left(\widetilde{S}_{g(\alpha)}\right)$ is a continuous mapping.

Therefore, we obtain a holomorphic mapping $\mathcal{E}_{\alpha}: \mathcal{Q}_{\mathcal{A}}(S) \rightarrow \mathcal{Q}_{\widetilde{\mathcal{A}}_{g(\alpha)}}\left(\widetilde{S}_{g(\alpha)}\right)$.

Proof of Theorem 1.3. Since $\alpha: \widetilde{S}_{g(\alpha)} \rightarrow S$ is a characteristic covering, then $\operatorname{Mod}(S) \subset \operatorname{Mod}\left(\widetilde{S}_{g(\alpha)}\right)$. From Lemma 3.1, it induces a continuous mapping $\widehat{\Phi}_{\alpha}: \widehat{M}(S)$ $\rightarrow \widehat{M}\left(\widetilde{S}_{g(\alpha)}\right)$. For every multicurve $\mathcal{A}$ on $S$, from Lemma 4.1, there exists a holomorphic mapping

$$
\mathcal{E}_{\alpha}: \mathcal{Q}_{\mathcal{A}}(S) \rightarrow \mathcal{Q}_{\widetilde{\mathcal{A}}_{g(\alpha)}}\left(\widetilde{S}_{g(\alpha)}\right)
$$

then

$$
\widehat{\Phi}_{\alpha}: \bigcup_{\mathcal{A C S}}\left(\mathcal{Q}_{\mathcal{A}}(S) / \operatorname{Mod}(S)\right) \rightarrow \bigcup_{\mathcal{A} \subset \mathcal{S}}\left(\mathcal{Q}_{\alpha^{-1}(\mathcal{A})}\left(\widetilde{S}_{g(\alpha)}\right) / \operatorname{Mod}\left(\widetilde{S}_{g(\alpha)}\right)\right)
$$

is a holomorphic mapping. Therefore, $\widehat{\Phi}_{\alpha}: \widehat{M}(S) \rightarrow \widehat{M}\left(\widetilde{S}_{g(\alpha)}\right)$ is a holomorphic mapping.

Definition 4.2. From the directed set $\operatorname{Mor}\left(\mathcal{C}^{c h}(S)\right)$, the direct limit of augmented moduli spaces over $S$ is defined as follows:

$$
\widehat{M}_{\infty}(S):=\operatorname{dir} . \lim . \widehat{M}\left(\widetilde{S}_{g(\alpha)}\right), \quad \alpha \in \operatorname{Mor}\left(\mathcal{C}^{c h}(S)\right),
$$


which is called the universal commensurability augmented moduli space.

Corollary 4.3. The universal commensurability augmented moduli space $\widehat{M}_{\infty}(S)$ has a complex orbifold structure.

Next, we show that the subgroup $\operatorname{Caut}\left(\pi_{1}(S)\right)$ acts on $\widehat{T}_{\infty}(S)$ to produce $\widehat{M}_{\infty}(S)$ as the quotient.

Proof of Theorem 1.4. The directed system $\operatorname{Mor}\left(\mathcal{C}^{c h}(\widehat{T}(S))\right)$ of augmented Teichmüller spaces is the cofinal sub-system of $\operatorname{Mor}(\mathcal{C}(\widehat{T}(S)))$ and set $\widehat{T}_{\infty}^{c h}(S)$ the corresponding direct limit space. The inclusion of $\mathcal{C}^{c h}(S)$ in $\mathcal{C}(S)$ induces a natural homeomorphism of $\widehat{T}_{\infty}^{c h}(S)$ onto $\widehat{T}_{\infty}(S)$. Clearly, it follows from the definition of the subgroup Caut $\left(\pi_{1}(S)\right)$ that Caut $\left(\pi_{1}(S)\right)$ acts on $\widehat{T}_{\infty}^{c h}(S)$ to produce $\widehat{M}_{\infty}(S)$ as the quotient. Therefore, identifying $\widehat{T}_{\infty}^{c h}(S)$ with $\widehat{T}_{\infty}(S)$ by the above homeomorphism, we obtain the result.

Acknowledgements. We thank the referees for many important and useful comments.

\section{References}

[1] AвıкоғF, W.: Augmented Teichmüller spaces. - Bull. Amer. Math. Soc. 82, 1976, 333-334.

[2] Abikoff, W.: Degenerating families of Riemann surfaces. - Ann. of Math. (2) 105, 1977, $29-44$.

[3] Biswas, I., Mitra, M., and S. Nag: Thurston boundary of the Teichmüller spaces and the commensurability modular group. - Conform. Geom. Dyn. 3, 1999, 50-66.

[4] Biswas, I., and S. NAG: Weil-Petersson geometry and determinant bundles over inductive limits of moduli spaces. - Contemporary Math. 211, 1997, 51-80.

[5] Biswas, I., S. Nag, and D. Sullivan: Determinant bundles, Quillen metrics and Mumford isomorphisms over the universal commensurability Teichmüller space. - Acta Math. 176, 1996, $145-169$.

[6] Deligne, P., and D. Mumford: The irreducibility of the space of curves of given genus. Inst. Hautes Etudes Sci. Publ. Math. 36, 1969, 75-109.

[7] Harvey, W.: Chabauty spaces of discrete groups. - In: Discontinuous groups and Riemann surfaces, vol. 79, 1974, 239-246.

[8] Hu, G., and Y. QI: The isometric embedding of the augmented Teichmüller space of a Riemann surface into the augmented Teichmüller space of its covering surface. - Kodai Math. J. 42, 2019, 376-392.

[9] Hubbard, J., and S. Koch: An analytic construction of the Deligne-Mumford compactification of the moduli space of curves. - J. Differential Geom. 98, 2014, 261-313.

[10] Kahn, J., and V. Markovic: The good pants homology and the Ehrenpreis Conjecture. Ann. of Math. (2) 182, 2015, 1-72.

[11] Kra, I.: Canonical mappings between Teichmüller spaces. - Bull. Amer. Math. Soc. 4, 1981, $143-179$.

[12] Odden, C.: The baseleaf preserving mapping class group of the universal hyperbolic solenoid. - Trans. Amer. Math. Soc. 357, 2005, 1829-1858.

[13] Penner, R. C., and ŠArić, D.:, Teichmüller theory of the punctured solenoid. - Geom. Dedicata $132,2008,179-212$.

[14] ŠARIĆ, D.: Earthquakes and Thurston boundary for the Teichmüller space of the universal hyperbolic solenoid. - Pacific J. Math. 233, 2007, 205-228. 
[15] ŠARIĆ, D.: On quasiconformal deformations of the universal hyperbolic solenoid. - J. Anal. Math. 105, 2008, 303-343.

[16] ŠARIĆ, D.: The Teichmüller theory of the solenoid. - In: Handbook of Teichmüller theory, vol. II, 2009, 811-857.

[17] Shafarevich, I. R.: On some infinite-dimensional groups II. - Math. USSR Izvest. 18, 1982, $185-194$.

Received 23 May $2020 \bullet$ Accepted 8 January 2021 